\title{
Percepción de Funcionalidad Familiar y Trauma Infanto- juvenil: un estudio cualitativo
}

\section{Perception of Family Functioning and Child-Adolescent Trauma: A Qualitative Study}

Ramón Florenzano, Isabel González, Paula Errázuriz, Raúl Ventura, Allister Gibbons, Bernardita Blümel, Carolina Muñiz, Carolina Marty, Kristina Weil y Cesar Carvajal.*

\begin{abstract}
Resumen
Se comparan las características de quince mujeres hospitalizadas en un hospital general publico en Santiago de Chile, que recuerdan más de tres experiencias traumáticas antes de los 16 años, con 15 casos que no recuerdan ninguno. En los 30 casos se estudian características socio-demográficas, y se aplican las siguientes escalas: Acontecimientos Traumáticos de Perry (TAI), las escalas de percepción familiar de Valdés y Cols (CTF-CSF) y la Escala Abreviada de Conductas de Riesgo (ECRA). Se comparan ambos grupos, constatándose una mayor frecuencia de dificultades en las relaciones interpersonales, disfuncionalidad familiar y conductas de riesgo en las mujeres con antecedentes anamnésticos de polipsicotrauma antes de los 16 años. Se concluye insistiendo en la importancia de la prevención de la violencia intrafamiliar y de los tratamientos adecuados para las víctimas.
\end{abstract}

\begin{abstract}
This paper compares characteristics of fifteen women hospitalized in public hospitals in Santiago de Chile who recalled more than three traumatic events before age 16, with a matched group of 15 women without recollection of child trauma. The thirty cases were interviewed collecting socio-demographics, and the following scales: Perry's Traumatic Events (TAI); family perception scales of Valdes et al (CTF-CSF), and a short risk behavior scale (ECRA). The comparison found more interpersonal difficulties, family disfunction and risk behaviors among women with more recollection of multiple psychic traumas prior to age 16 . We end underscoring the importance of prevention of family violence and the need of adequate treatment for victims.
\end{abstract}

* Servicio de Psiquiatría Hospital del Salvador, Facultad de Medicina Universidad de los Andes Facultad de Psicología Universidad del Desarrollo. email: rflorenzano@gmail.com 


\section{Introducción}

El impacto de las experiencias traumáticas vividas en la infancia en el desarrollo de la organización psíquica adulta ha sido un tema relevante dentro de la búsqueda de la etiología de patologías mentales desde antiguo. Los descubridores del método pl psicoanalítico, Freud y Breuer plantearon una hipótesis etiológica sobre las neurosis centrada en experiencias traumáticas tempranas. En la concepción inicial de Freud (1890-97) con respecto a la etiología de las neurosis, el trauma es considerado un suceso externo acaecido en la infancia del sujeto, que no pudo ser elaborado por su gran carga afectiva, perdurando en forma de recuerdo, a modo de un cuerpo extraño que puede seguir produciendo síntomas en el presente. Posteriormente, en lo que Laplanche y Pontalis llaman "el giro", Freud abandonó esta teoría, al afirmar, como lo resume Del Río $\left({ }^{1}\right)$ "que los sintomas neuróticos no estaban directamente relacionados con acontecimientos reales sino con fantasías derivadas del deseo y que en lo que concierne a las neurosis, la realidad psíquica tiene más importancia que la realidad material". Los síntomas neuróticos pasan a ser entendidos no como una consecuencia de eventos traumáticos reales ocurridos en la infancia, sino por la represión propia de la sexualidad infantil. Freud vuelve al tema del efecto del trauma después de la Primera Guerra Mundial, al estudiar sueños de pacientes que sufrían neurosis de guerra, los cuales no eran realizaciones de deseo sino que repetición de las vivencias traumáticas con los concomitantes afectivos de pena y dolor. El trauma, de origen interno o externo, produce aumentos de flujo de energía que superan la capacidad de ligadura, amenazando la integridad del sujeto. La mente, para ligar esta energía excesiva, vuelve a la situación traumática. Luego, dicho autor volvió a elaborar en forma más compleja su teoría inicial en "Inhibición, Sintoma y Angustia" ( $\left.{ }^{2}\right)$, al mostrar como el Yo en el modelo estructural tramita las amenazas de desborde en forma anticipatoria mediante diversos mecanismos defensivos. En esta etapa Freud considera indiferente el hecho de que las amenazas para el Yo surjan desde dentro o desde fuera. Posteriormente, Ferenczi, en "La confusión de lenguas entre los adultos y el niño" vuelve a insistir en la importancia de los factores traumáticos externos en la patogenia de las neurosis. Al modificar su técnica, los pacientes volvieron a relatar experiencias traumáticas sobre todo de abuso sexual. $\left({ }^{3}\right)$.

Dentro de las teorías psicoanalíticas mas recientes, basadas en el estudio de las patologías de los adultos, diversos autores como Sullivan, Bowlby, Winnicott y Kohut, consideran la conducta real de la madre hacia el bebé como crucial en la reconstrucción de la historia de este último $\left({ }^{4}\right)$. Del Río, en nuestro medio, desde una perspectiva winnicotiana, parte de la dependencia absoluta del bebé de su madre, y de la importancia de la mediación del medio ambiente: cuando este falla, se da lugar a ansiedades catastróficas y la continuidad del existir se interrumpe. Su definición es: "el trauma es una intrusión que proviene del ambiente y de la reacción del individuo a este, sobrevenida antes de que el individuo desarrolle los mecanismos que hacen predecible lo impredecible". En la medida que esta intrusión dificulta la representación psíquica del deseo, se detiene el desarrollo en afectos difusos y en emergencia de síntomas somáticos $\left({ }^{5}\right)$.

Autores post racionalistas, como Crittenden (1997) proponen que los niños internalizan información al interactuar con sus cuidadores y formando así el esquema base de sus futuras relaciones. Propone que los individuos cuyo tipo de vínculo se caracteriza por una visión negativa de sí mismo guían sus conductas por sus afectos más que por sus cogniciones por lo que estarían incapacitados para regular sus afectos teniendo una alta fragilidad de sus sistemas emocionales. Además de las teorías acerca del trauma vivido en la infancia y sus efectos en el 
desarrollo psicológico, diversos investigadores se han preocupado por estudiar empíricamente las consecuencias psicológicas de haber sido victima de experiencias traumáticas durante la infancia mediante trabajos. Numerosas investigaciones han relacionado maltrato infantil tanto psicológico como físico, abuso sexual y/o negligencia parental con psicopatologías tales como trastorno de somatización, depresión, riesgo suicida, impulsividad y trastornos de la personalidad. Dichas investigaciones coinciden con la hipótesis que establece que el número de experiencias traumáticas, su clase, severidad, cronicidad y la etapa vital de la victimización son factores predictores de consecuencias nocivas para la salud mental adulta $(6,7,8,9,10)$.

Algunos investigadores han establecido ciertos factores de riesgo para sufrir consecuencias severas, tales como: que el evento traumático sea generado en la infancia, infligido por seres humanos, por una figura de apego o a cargo de su cuidado, con crueldad y de manera crónica. La exposición a experiencias estresantes en la infancia y adolescencia afectaría en mayor o menor grado la adquisición para la vida adulta de capacidades yoicas claves como la autorregulación emocional, la confianza básica, el pensamiento reflexivo e incluso la salud física $(11,12,13,14)$. Además se ha postulado que el abuso y la negligencia infantil causan una disrupción en la identificación, comunicación, procesamiento cognitivo y elaboración de los afectos $\left({ }^{15}\right)$. Al mismo tiempo, en la experiencia clínica se observa que pacientes con patologías serias de la personalidad presentan como recuerdos anamnésticos, disfunción familiar de carácter grave, inconstancia, inversión de roles, descuido, duelos, violencia física intrafamiliar o abuso sexual $\left(16,17,{ }^{18}\right)$. Entre las investigaciones realizadas a nivel comunitario con grandes muestras de población general se ha encontrado una correlación entre maltrato, abuso y negligencia en la infancia con comorbilidad psiquiátrica. Si bien estos estudios transversales no pueden establecer relaciones causales entre ambas variables, si han podido llegar a conclusiones tales como: que la historia de abuso físico es tan importante en su correlato de comorbilidad psiquiátrica como el haber sufrido abuso sexual en la infancia, o, que existen ciertas diferencias de género en la asociación entre trauma infantil y patología adulta $\left({ }^{19}\right)$.

En nuestro medio, el maltrato infantil y sus consecuencias para la salud del sujeto han adquirido relevancia en la medida que recientes estudios han develado la real prevalencia de este problema en la población infantil. Así la UNICEF y el Departamento de Psicología de la Universidad de Chile ${ }^{20}$ ) establecieron para una muestra extrapolable a todo el país, que el $63 \%$ de los niños encuestados aseveró haber recibido algún tipo de violencia física por parte de un progenitor, con un 14,5\% adicional que afirmó haber recibido algún tipo de violencia psicológica. Otro de los acercamientos nacionales a la evaluación de los estragos causados por el maltrato infantil lo realizaron Nuhmhauser y Schwartz, quienes evaluaron a mujeres consultantes en la Unidad de Salud Mental del Hospital de Ancud, con polisintomatología tanto psiquiátrica como física, encontrando una serie de factores sociales actuales que se entremezclan con una amplia carga traumática infantil y de factores predisponentes. Dichos factores configuran un cuadro donde se revelan la coexistencia de diversos cuadros psiquiátricos entre los cuales están trastornos somatomorfos, depresión y estrés $\left({ }^{13}\right)$.

Otros intentos de aproximación al tema son los realizados por nuestro grupo de investigación $(12,15)$. En una primera etapa nos dedicamos a establecer la frecuencia de antecedentes de trauma psíquico infantil en mujeres hospitalizadas, para posteriormente, estudiar la relación entre recuerdo de trauma psíquico infantil y la presencia de tras- 
tornos somatomorfos junto a trastornos de la personalidad en una población de pacientes hospitalizados. En dichos estudios se descubrió una correlación estadísticamente significativa entre frecuencia de trauma y el trastorno de somatización, así como con trastorno límite de personalidad. En estudios posteriores nos hemos centrado en los aspectos positivos y de protección en relación al trauma, en especial en la capacidad de recuperación mostrada por individuos, familias y comunidades resilientes $\left({ }^{21}\right)$

Con el fin de examinar los efectos que tienen la experiencias traumáticas infanto juveniles, en el presente trabajo se compara un grupo de mujeres que recuerdan experiencias traumáticas con casos que no recuerdan ninguno, evaluándose las relaciones interpersonales, funcionalidad familiar y conductas de riesgo. Este estudio con foco cualitativo, se enmarca dentro de un proyecto de investigación mayor subsidiado por la Dirección de Investigación de la Universidad de Los Andes. Se buscó, a través de un estudio censal en servicios clínicos de cuatro hospitales generales, determinar la prevalencia de psicopatología en pacientes hospitalizados, así como la prevalencia de acontecimientos traumáticos ocurridos antes de los 16 años de edad, comparando frecuencias de psicopatología y severidad del trastorno de personalidad con el fin ulterior de diseñar un modelo de intervención para prevención secundaria de la aparición del estrés post traumático.

\section{Objetivos}

El objetivo general fue determinar las características de las relaciones interpersonales y conductas de riesgo, así como la percepción del funcionamiento familiar de la familia de origen y familia actual, en mujeres usuarias del sistema público de salud, que presenten recuerdos de trauma infanto-juvenil en comparación con otras que no manifiesten estos recuerdos.
Otro propósito de la investigación fue conocer las características de los eventos traumáticos recordados por las mujeres y el impacto que éstas consideran que tuvo en su vida. Se esperaba encontrar que las pacientes que tuvieran recuerdo anamnéstico frecuente de psicotraumas infanto-juveniles, presentaran una peor calidad de las relaciones interpersonales, junto con una percepción de mayor disfuncionalidad familiar en su familia de origen y familia actual, y una mayor cantidad de conductas de riesgo. También se esperaba que las mujeres con antecedentes traumáticos consideraran que éstos tuvieron un impacto alto en sus vidas.

\section{Metodología}

Durante los meses de Junio, Julio, Octubre y Diciembre del año 2003 se entrevistaron 504 mujeres usuarias de cuatro hospitales, tres en la Región Metropolitana y otro en la VII Región. La mayoría de ellas se encontraban hospitalizadas en un hospital público de la Región Metropolitana al momento de ser entrevistadas. El proyecto fue aprobado por el Comité de Ética de dicho hospital, y la entrevista se realizó después de obtener consentimiento informado. Se aplicó la Escala de Trauma de Marshall (22) a fin de establecer el número de eventos traumáticos que recordaban las mujeres. Por un muestreo sucesivo se seleccionaron 15 casos que presentaban recuerdo de psicotrauma frecuente (tres o mas en la escala de Marshall) y 15 casos sin ningún recuerdo. Cuando el entrevistado presentaba puntajes cero o tres o más se procedió a administrar los siguientes instrumentos:

1) Cuestionario CSF (Como es Su Familia), validado en Chile por Valdés et al $\left({ }^{23}\right)$, utilizado para establecer la percepción de funcionamiento familiar.

2) Escala Abreviada de Conductas de Riesgo de Blum et $\mathrm{Al}\left({ }^{24}\right)$ validada en Chile por Florenzano et al $\left({ }^{25}\right)$. 
3) Escala de Acontecimientos Traumáticos de Perry $\left({ }^{26}\right)$, que permite una indagación en mayor profundidad de los eventos traumáticos vivenciados por el paciente.

En el procesamiento de la información, se creó una base de datos que fue analizada mediante el programa estadístico SPSS en su versión 11.0. Primero se efectuó un análisis simple de comparación de razones a partir de los datos tabulados.

\section{Resultados $^{27}$}

\section{Descripción características del grupo de mujeres con trauma}

La tabla 1 resume estos resultados: de los 15 casos con antecedente de trauma antes de los 16 años, encontramos que diez mujeres informan separación traumática del padre, madre o persona que la cuidaba por más de un mes. En la mayoría de estas mujeres (7/ 10) la principal persona a su cuidado eran el padre, la madre o ambos. Más de la mitad de las mujeres $(6 / 10)$ con este antecedente de trauma reportan fallecimiento en la familia inmediata antes de los 16 años.

Casi todas las mujeres con antecedentes de trauma (13/15) tuvieron la experiencia de haber sufrido algún castigo físico importante. El castigo físico era algo común en la vida de la mayoría de ellas (9/13), ya que la forma habitual para disciplinar a los niños en sus casas era a través de golpes. Incluso en el hogar de algunas de estas mujeres (5/13) el golpe con objeto era una de las formas habituales de disciplinar. De las 13 mujeres que recibieron castigo físico, más de la mitad (8/ 13) quedaron alguna vez con un daño físico como consecuencia de un castigo físico.

Más de dos tercios de las mujeres (11/15) dicen haber presenciado violencia física entre los padres o personas que la cuidaban. La mayoría de estas mujeres (9/11) reporta que el presenciar violencia en su casa ocurría "frecuentemente", lo que da cuenta de que no eran hechos aislados.

Diez de las 15 mujeres afirman haber tenido contacto sexual forzado con un niño, adolescente o adulto no familiar, o un contacto sexual forzado con un pariente. En cuanto a las 8 mujeres que tuvieron contacto sexual forzado con un pariente, 3 de 5 consideran que el incidente en ese momento la alteró mucho y 2 de 5 extremadamente. En cuanto al efecto que tuvo en sus vidas 4 de 5 responden que fue alto. No hay ningún caso registrado que reporte haber intentado obtener ayuda a partir de una experiencia sexual involuntaria.

Al preguntar a las mujeres qué aconsejaría a los demás basándose en sus experiencias, las respuestas que más se repiten son: contar a otros lo que le ocurre y pedir ayuda.

\section{Comparación entre el grupo de mujeres que recuerdan politrauma con quienes no lo tienen.}

\section{Familia de origen e infancia}

La presencia de la madre en la infancia es muy similar entre ambos grupos (10 de 15 en los pacientes sin trauma y 11 de 15 en las pacientes con trauma). Sin embargo, hay una diferencia importante en relación a los padres. Este se encuentra presente en la infancia de la mayoría de las pacientes sin trauma (12 de 15) y en menos de la mitad de las pacientes con antecedentes de trauma (6 de 15).

Al considerar la relación con los padres en la infancia, tanto el padre como la madre son percibidos como más afectuosos en las mujeres sin trauma (padre 9/14, madre 10/ 13) que en las mujeres con trauma (padre $3 /$ 14, madre 5/14). En cuanto al afecto en la familia de origen 13 de 15 mujeres sin trauma consideran que este era confiable y consistente en comparación a 11 de 15 de las mujeres con trauma. 
En relación a la disciplina familiar, el grupo sin trauma refiere en mayor número que las normas en la familia eran claras y consistentes. Sólo cuatro de las 15 mujeres del grupo con trauma refieren que las normas en sus casas eran generalmente justas, en comparación a 12 de las 15 en el grupo sin trauma.

Existe una gran diferencia en la frecuencia de los castigos recibidos entre ambos grupos. El grupo sin antecedentes de trauma dice haber sido castigadas en promedio menos de una vez al mes $(0,8)$, mientras que las mujeres con antecedentes de trauma en promedio dijeron haber sido castigadas 21,8 veces.

En cuanto a la forma habitual para disciplinar a los niños, las mujeres con recuerdo de trauma fueron disciplinadas de forma más agresiva que el grupo sin estos antecedentes. La forma más habitual de disciplina en ambos grupos fue el reto, (12/15 en ambos grupos), seguido por la negación de permisos en más de un tercio de las mujeres, con igual frecuencia en ambos grupos (6/15). La palmada fue usada tres veces mas en el grupo con antecedentes de trauma que en el grupo sin antecedentes (9/15 versus 3/15). El abuso verbal no fue usado en las mujeres sin trauma $(0 / 15)$, pero fue usado en la mayoría de las mujeres con trauma $(9 / 15)$. Similar proporción se encuentra en el golpe como forma habitual de disciplinar, ya que sólo una mujer sin antecedente de trauma lo reporta, versus 9 del grupo con antecedentes de trauma. Por último, el golpe con objeto fue cinco veces más frecuente en el grupo de mujeres con trauma (1/15 versus $5 / 15)$.

Al preguntarle a las mujeres sobre la forma usual en que los padres resolvían sus desacuerdos, como se muestra en la tabla 2, el grupo con antecedentes traumáticos responde en mayor número que las mujeres sin antecedentes traumáticos, que sus padres: discutían (9/15 versus $7 / 15)$, gritaban (8/15 versus 3/15), amenazaban con golpear a la otra persona (5/15 versus $1 / 15)$, rompían o arrojaban cosas $(7 / 15$ versus $1 / 15)$, golpeaban (3/15 versus 0/15). En el grupo de mujeres sin recuerdos de trauma, fue más frecuente la respuesta "nunca vio a los padres enojados o peleando" (10/15 versus 6/15).

Auto descripción y aspectos resilientes. La tabla 3 muestra que la mayoría de las mujeres, con y sin trauma, consideran que fueron "especiales" para alguien durante su infancia (12/ 15 sin trauma y 11/ 15 con trauma). Al preguntarles a las mujeres si eran talentosas (en deportes, pasatiempos, colegio o actividad creativa) la mayoría de las mujeres de ambos grupos responden afirmativamente (11/15 sin trauma versus 10/15 con trauma).

En relación a aspectos positivos y resilientes: el grupo de mujeres sin trauma se describen a sí mismas durante su infancia de forma más positiva que las mujeres con antecedentes de trauma. El primer grupo se describe con mayor frecuencia como felices (12/ 15 versus $7 / 15)$, amistosas (11/15 versus $3 / 15)$, entusiastas $(9 / 15$ versus $3 / 15)$ y animosas (8/15 versus 2/15). Al mismo tiempo, el grupo de mujeres con antecedentes de trauma se describen más a sí mismas como tristes $(10 / 15$ versus $3 / 15)$, solitarias $(9 / 15$ versus $4 / 15)$, asustadas $(7 / 15$ versus $2 / 15)$ y tímidas (7/15 versus $2 / 15)$.

En relación a la descripción del grupo de pares en la infancia, en los casos de mujeres sin antecedentes de trauma, más de la mitad (8/15) dicen haber pertenecido a un grupo positivamente identificado, versus menos de un tercio (4/15) de aquellas con antecedentes de trauma. Dos mujeres con antecedentes de trauma dicen haber pertenecido a un grupo negativamente identificado, mientras que en el grupo sin antecedentes de trauma no hay ninguna. Se mantiene la tendencia en la adolescencia a que las mujeres sin trauma pertenezcan o estén al margen de un grupo identificado positivamente más que las con trauma y estén menos asociadas (pertenecer 
o estar al margen) a grupos identificados negativamente que las con trauma.

Sólo 2 de los 15 casos de mujeres sin antecedentes de trauma intentó huir del hogar comparando con 9 de 13 casos de mujeres con antecedentes de trauma.

\section{Situación actual}

La tabla 4 muestra la situación actual de las pacientes entrevistadas. En cuanto a la relación con los hermanos, el grupo sin antecedentes de trauma dice tener una relación cercana con éstos en 13 casos, versus sólo 8 del grupo con trauma. Este último grupo dice tener una relación distante en 4 casos, versus sólo uno del grupo sin trauma.

En cuanto al estado civil, las mujeres sin trauma se han casado en mayor proporción que aquellas con trauma (9/15 versus 6/14). La convivencia es mayor en mujeres con trauma. El número de solteras y separadas es igual en ambos grupos.

Al revisar la comunicación conyugal de las mujeres con sus parejas, se puede observar que esta es mejor en las pacientes que no recuerdan antecedentes de trauma. Las mujeres sin trauma consideran que en su familia el padre con mayor frecuencia dedica tiempo cada día para hablar con los hijos; con mayor frecuencia les satisface la conversación con su esposo o pareja; con mayor frecuencia les es fácil expresar sus sentimientos a su esposo y pareja; con mayor frecuencia sienten que su esposo o pareja las entiende; y con mayor frecuencia cuando están en dificultades lo comentan con esposo y pareja.

En cuanto a la jerarquía en el hogar, el triple de mujeres sin recuerdo de trauma, en relación a las con trauma, dicen que tanto el padre como la madre mandan en su familia (6/15 versus 2/15). En cuanto a la comunicación y conexión de la madre y la familia nuclear, las mujeres sin antecedentes de trauma con mayor frecuencia que las mujeres con antecedentes de trauma dicen: turnarse las tareas y responsabilidades de la casa; cada uno expresa lo que desea o piensa; la madre dedica tiempo, cada día para hablar con los hijos; comparten alguna comida cada día; se reúnen en algún momento del día para conversar y / o compartir alguna actividad. Las mujeres con trauma, eso sí, dicen que con más frecuencia les gusta pasar juntos el tiempo libre que las mujeres sin trauma.

En relación a las conductas de riesgo, las mujeres con antecedentes de trauma presentan más conductas de riesgo que las mujeres sin estos recuerdos. Las conductas de riesgo evaluadas fueron: fumar, embriagarse, consumo de marihuana, uso de drogas (cocaína, heroína, L.S.D.), otras drogas (tranquilizantes, hipnóticos, etc.), uso de solventes volátiles (neopren u otro), haber estado involucrada en pelea o riña, haber intentado matarse o haber quedado embarazada sin desearlo. Las mujeres con trauma presentan una mayor incidencia de todas las conductas de riesgo nombradas. Aqupellas en que se aprecian mayores diferencias entre ambos grupos son: el uso de drogas como tranquilizantes, hipnóticos, etc. (sin trauma $2 / 15$ versus con trauma 10/ 15), haber estado involucrada en riñas o peleas ( $\sin$ trauma $1 / 14$ versus con trauma 8 / 15) e intentos de suicidio ( $\sin$ trauma $2 / 15$ versus con trauma 11/15).

\section{Conclusiones}

A partir de los resultados sobre el grupo de mujeres entrevistadas, se confirma la hipótesis de que las mujeres con recuerdo de trauma infanto juvenil presentan una peor calidad de relaciones interpersonales, junto a una percepción de mayor disfuncionalidad familiar en su familia de origen y familia actual y una mayor cantidad de conductas de riesgo. La mayoría de las mujeres considera que las situaciones traumáticas que vivieron tuvieron un alto impacto tanto en el momento de producirse éstas, como en el resto de sus vidas. 
Las mujeres con antecedentes de trauma tuvieron en su infancia familias más disfuncionales. Además de las experiencias traumáticas vividas dentro del hogar, estas mujeres vivieron situaciones familiares difíciles que muchas veces están relacionadas directamente con el maltrato intrafamiliar. En otros casos, situaciones extrafamialiares, por ejemplo el contacto sexual forzado con niño mayor o adulto no familiar, constituyen agravantes adicionales.

La mayor ausencia del padre y percepción de ambos padres como menos afectuosos da cuenta de la desventaja en cuanto al afecto recibido. Debido a que recibieron más castigo por parte de los padres, los cuales a su vez utilizaban formas de disciplinar más agresivas y resolvían entre sí los desacuerdos de manera más inadecuada y agresiva, constantemente estuvieron expuestas a altos niveles de agresión. Todo lo anterior daría cuenta de menos contención dentro del hogar y la vivencia de éste como un lugar hostil, lo que podría explicar porqué han tratado de huir más del hogar las mujeres con antecedentes que aquellas sin.

Se pudiera postular que el estar expuesto a relaciones agresivas, y a su vez, tener pocas figuras contenedoras dificulta la introyección de objetos buenos y con ello la posibilidad de establecer relaciones interpersonales constructivas a futuro. A su vez, el ser víctimas de la agresión o falta de cuidados de otros, hace percibir el mundo como hostil, disminuyendo la posibilidad de confiar en la bondad de los objetos tanto internos como externos.

El daño que queda a nivel de relaciones interpersonales se hace patente al ver la situación actual de las mujeres con antecedentes traumáticos, ya que estas tienen peor relación con sus hermanos carnales y con sus parejas. El poder conversar, sentirse comprendidas, expresar sus sentimientos y comentar dificultades les es más difícil a estas mujeres y sus familias que el grupo de las mujeres sin antecedentes traumáticos. Además, muestran tener familias más disfuncionales en la actualidad.

Las mujeres sin antecedentes de trauma logran hacer vidas más placenteras y con familias más funcionales. Esto podría ser explicado según lo revisado en el marco teórico, en tanto la exposición a relaciones negligentes y agresivas con los cuidadores parece favorecer la alexitimia, y con ello, interferir la capacidad para regular las emociones y establecer relaciones interpersonales satisfactorias. Sin embargo, cabe destacar que esta sería una de las múltiples variables que pueden ser abordadas para explicar las dificultades interpersonales observadas en las mujeres estudiadas.

La gran cantidad de conductas de riesgo realizadas por las mujeres con antecedentes de trauma da cuenta de su disminuida capacidad de autocuidado y de una mayor disposición a realizar conductas autodestructivas. El haber vivido experiencias traumáticas en su hogar, lugar donde deberían haberse sentido cuidadas, y en muchos casos, el que los responsables de estos situaciones traumáticas hayan sido sus cuidadores, parece haber dañado su capacidad para cuidarse a sí mismas. La impulsividad que está detrás de muchas de estas conductas de riesgo permiten pensar que las experiencias traumáticas dañan la capacidad de simbolización, pasando a la acción sin la previa reflexión sobre las consecuencias. Esto viene a graficar en cierta medida lo mencionado dentro del introducción teórica de este trabajo, en tanto se considera que la exposición a experiencias estresantes en la infancia afectaría la adquisición para la vida adulta de capacidades yoícas claves como la autorregulación emocional, la confianza básica, el pensamiento reflexivo e incluso la salud física, déficit que incidiría en la capacidad de autocuidado. 
Como se puede observar a través de esta investigación, el impacto de haber sufrido traumas durantes la infancia y adolescencia deja huellas extremadamente dañinas y duraderas en sus víctimas. En los resultados se hace patente lo expuesto en la introducción teórica de cómo las experiencias traumáticas tienen un impacto importante en la organización psíquica adulta.

Fonagy y Target $\left({ }^{28}\right)$ señalan que la visión psicoanalítica clásica ha destacado la experiencia intrapsíquica del individuo y se ha desinteresado relativamente del "mundo real" en el cual las experiencias intrapsíquicas se desarrollaron. Hay así una suposición silenciosa de que las etapas madurativas de las pulsiones son más importantes que los así llamados "accidentes" del medio ambiente. En contraste, teorías mas recientes, basadas en el estudio de las patologías de los adultos, ven la conducta real de la madre hacia el bebé como cruciales en la reconstrucción de la historia de este último Una controversia actual en psicoanálisis (la cual refleja un debate cultural) tiene que ver con la "cognoscibilidad" de la experiencia temprana. Shengold (1989) relaciona esta controversia con el debate iniciado en el siglo XVIII entre George Berkeley acerca de la cognoscibilidad de la realidad más allá de la mente y de sus ideas. En 1977 Florence Rush argumentó que Freud había tanto descubierto como encubierto la extensión del abuso sexual infantil (1977). Masson (1984) criticó a Freud por haber defensivamente abandonado y deliberadamente ocultado evidencia que apoyaba la teoría de la seducción en las neurosis. En realidad, Freud nunca "suprimió" la teoría de la seducción sino que la acomodó para hacerla corresponder a los hechos, y trajo esta relación con el descubrimiento de la sexualidad infantil y su potencial de patogénesis (Hanley, 1997). En 1906 Freud insistió que los 18 pacientes de su "Etiología de la Histeria" (Freud, 1906) le habían dado descripciones exactas de haber sido seducidas en la niñez. Freud reforzó su idea del poder patogénico de la experiencia real de seducción en sus "Conferencias Introductorias" (Freud, 1916-17), en "Sexualidad Femenina" (Freud, 1931 ${ }^{\mathrm{a}}$ ) y en "Moisés y el Monoteísmo" (Freud 1939). En contraste, los psicoanalistas que adoptan un apostura hermenéutica (Steele, 1979, Spence, 1982), repudian la búsqueda terapéutica de un pasado "real" y afirman el criterio de la coherencia interna como el único test apropiado de "verdad". Spence (1982); insiste en que el psicoanálisis no puede pretender un acceso privilegiado al conocimiento del pasado (lo que implicaba la noción de Freud de una labor cuasi-arqueológica). Argumenta este autor que el encuentro con el pasado en el contexto terapéutico es un acto de creación de "una narración coherente y plausible en la vida de nuestro paciente". Este autor hace una advertencia crítica: “Una vez establecida, la verdad narrativa se hace parcialmente verdad; en la medida que se repite y extiende, se hace familiar, y su familiaridad aumenta su plausibilidad, terminando completamente cierta".

Ya que la gran mayoría de las situaciones traumáticas se dan dentro del hogar, se hace urgente intensificar el trabajo en prevención del maltrato intra familiar. A su vez, se requiere investigar e implementar tratamientos psicológicos que ayuden, dentro de lo posible, a reparar el daño sufrido por las víctimas. 
Tabla 1 Características comparativas de pacientes con trauma masi vo versus controles, 30 mujeres hospitalizadas en Servicio de Psiquiatría Hospital del Salvador (SPHDS), 2004

\begin{tabular}{|lrrrr|}
\hline & Marshall 3+ & Porcentaje & Marshall 0 & Porcentaje \\
Madre presente en casa & 11 & 73.3 & 10 & 66.7 \\
Padre presente & 6 & 40.0 & 12 & 80.0 \\
Padres afectuosos & 3 & 21.4 & 9 & 64.3 \\
Madres afectuosas & 5 & 35.7 & 10 & 76.9 \\
Afecto confiable y consistente & 11 & 73.3 & 13 & 86.7 \\
Normas disciplina justas & 4 & 26.7 & 12 & 80.0 \\
Promedio castigos & 21.8 & & $0.8 /$ mes & \\
Reto como disciplina habitual & 12 & 80.0 & 12 & 80.0 \\
Negación permiso como disciplina & 6 & 40.0 & 6 & 40.0 \\
Palmada como disciplina & 9 & 60.0 & 3 & 20.0 \\
Abuso verbal & 9 & 60.0 & 0 & 0.0 \\
Abuso golpe & 9 & 60.0 & 1 & 6.7 \\
Golpe con objeto & 5 & 33.3 & 1 & 6.7 \\
\hline
\end{tabular}

TABLA 2 Cómo resuelven desacuerdos padres de 30 mujeres hospitalizadas en SPHDS , 2004

\begin{tabular}{|lrrrr|}
\hline & Marshall 3+ & Porcentaje & Marshall 0 & Porcentaje \\
Discusión & 9 & 60.0 & 7 & 46.7 \\
Gritos & 8 & 53.3 & 3 & 20.0 \\
Amenaza de Golpes & 5 & 33.3 & 3 & 20.0 \\
Arrojar cosas & 7 & 46.7 & 1 & 6.7 \\
Irse del Hogar & 1 & 6.7 & 0 & 0.0 \\
\hline
\end{tabular}


Tabla 3. Autodescripción de de 30 mujeres hospitalizadas en SPHDS , 2004

\begin{tabular}{|lrrrr|}
\hline & Marshall 3+ & Porcentaje & Marshall 0 & Porcentaje \\
Fueron especial para alguien & 11 & 73.3 & 12 & 80 \\
Talento deportivo u otro & 10 & 66.7 & 11 & 73 \\
Infancia Feliz & 7 & 46.7 & 12 & 80.0 \\
Amistosas & 3 & 20.0 & 11 & 73.3 \\
Entusiastas & 3 & 20.0 & 9 & 60.0 \\
Animosas & 2 & 13.3 & 8 & 53.3 \\
Tristes & 10 & 66.7 & 3 & 20.0 \\
Solitarias & 9 & 60.0 & 4 & 26.7 \\
Asustadizas & 7 & 46.7 & 2 & 13.3 \\
Tímidas & 7 & 46.7 & 2 & 13.3 \\
Grupo de pares positivo & 4 & 26.7 & 8 & 53.3 \\
Grupo de pares negativo & 2 & 13.3 & 0 & 0.0 \\
Intento de fuga del hogar & 9 & 60.0 & 2 & 13.3 \\
\hline
\end{tabular}

Tabla 4 Situación actual familiar y personal de de 30 mujeres hospitalizadas en SPHDS , 2004

\begin{tabular}{|lrrrr|}
\hline & Marshall 3+ & Porcentaje & Marshall 0 & Porcentaje \\
Relacion con hermanos & 8 & 53.3 & 13 & 86.7 \\
E. civil casadas & 6 & 40.0 & 9 & 60.0 \\
Ambos padres mandan & 2 & 13.3 & 6 & 40.0 \\
Uso de drogas con tranquilizantes & 10 & 66.7 & 2 & 13.3 \\
Involucrada en riñas o peleas & 8 & 53.3 & 1 & 6.7 \\
Intentos de suicidio & 11 & 73.3 & 2 & 13.3 \\
\hline
\end{tabular}

Fecha Recepción Artículo: 13 de abril 2005

Fecha Evaluación Final: 22 de mayo 2006 


\section{Bibliografia}

1 Del Río, Marta. Trauma, un modelo de daño y su abordaje terapéutico. Revista APCH, 2000

2 Freud, S. Inhibición, Síntoma y Angustia. Londres, Hogarth. Standard Edition, 19:368, 1923

3 Ferenczi, S. La confusión de lenguajes entre los adultos y el niño. Int. J. Psychoan. 30: 225-230, 1933

4 Fonagy, P. y Target, M. BrunnerRoutledge, Psychoanalytic Theories: Perspectives from Developmental Psychopathology, Nueva York, 2003.

D.W: Winnicott. Escritor de Pediatría y Psicoanálisis. Paidos, Buenos Aires, 1999.

6 Florenzano R, Weil, K, Cruz C, Acuña J, Fullerton C, Muñiz C, Leighton C y Marambio M. Personalidad Limítrofe, somatización, trauma y violencia infantil: un estudio empírico. Rev Chil NeuroPsiquiat 2002: 40:335-340.

7 Bruce, A.; Stacey, H.; Scott, B.; Dea, R.; O’Connell, L.; Taylor, B. (1999) Childhood sexual abuse, Psychological distress and medical use among women. Psychosomatic Medicine 61;762-770

8 Johnson, J.; Cohen, P.; Brown, J.; Smailes, E.; Bernstein, D(1999). Childhoood maltreatment increased risk for personality disorders during early adulthood. Arch Gen Psychiatry 1999;56:600-606.

9 Cazabat, E, H (2002). Efectos del trauma prolongado en la infancia. Interpsiquis 2002 en www.psiquiatría.com

10 Florenzano, R, Weil, K, Acuña, J, Fullerton, C, Cruz Marín, C, Muñiz, C, Leighton, C.1 Personalidad Limítrofe, Somatización, Trauma y Violencia Infantil: Un nuevo estudio con muestra mayor. Revista Chilena de Salud, Mental, 149155, 2002.
11 Nuhmhauser, J.; Schwartz, N.(1999) Mujeres sobrepasadas: el drama médico social de las jefas de hogar politraumatizadas desde la infancia. Revisión clínica de 256 casos. Actas del Congreso Mundial de la Federación Mundial para la Salud Mental, Santiago de Chile, 1999.

12 Spatz, C.(1999) Childhood victimization and the development of personality disorders. Arch Gen Psychiatry vol 56, July 1999

13 Acuña, J; Fullerton, C.; Florenzano, R; Weil, K et cols (i?) Frecuencia de antecedentes de trauma psíquico infantil en mujeres hospitalizadas en dos hospitales generales de Santiago de Chile. ¿?

14 Brodsky, B.; Oquendo, M.; Ellis, S.; Haas, G.; Malone, K.; Mann, J. (2001) The relationship of childhood abuse to impulsivity and suicidal behavior in adults with mayor depression. Am J Psychiatry; 158:1871-1877

15 Zlotnick, Mattia, J., Zimmeumann, M., The relationship between post stress disorder, childhood trauma and alexitima in an outpatient sample, Journal of traumatic stress, 4: 25-32, 2001.

16 Marrone, M (2001). Teoría del Apego: un enfoque actual. Editorial Psimática. Buenos Aires, Argentina

17 Mares, M. (2000) Risk and preventive factors of post-traumatic stress disorder and its comorbid disorders. Current opinion in Psychiatry 2000, 13:587-589.

18 MacMillan, H.; Fleming,J.; Streiner, D.; Lin, E; Boyle, M.; Jamieson, E.; Duku, E.; Walsh, C. ; Wng, M. ; Beadsslee, W. (2001) Childhood Abuse and lifetime Psychopathology in a community sample. Am J Psychiatry; 158:1878-1883.

19 Walker, E; Gelfand, A; Katon, W; Koss, M; Von Korff, M; Bernstein, D; Russo, J (1999). Adult Health Status of Women with Histories of Childhood Abuse and Neglect. Am J Med 1999, 107: 332-339. 
Larraín, Soledad. Violencia Puertas Adentro: La Mujer Golpeada. Editorial Universitaria, Santiago de Chile, 1994.

21 Florenzano R y Valdes M. El Adolescente y sus Conductas de Riesgo. Tercera Edición, Ediciones Universidad Católica de Chile, Santiago 2005.

${ }^{22}$ Marshall, R.D., Schneier, F.R:, Lin, S.H., Simpson, H.B., Vermes, D y Liebowitz, M. Childhood Trauma and Dissociative Symptoms in Panic Disorder. Am. J. Psychiatry, 157: 451-453, 2000.

23 Valdés, M. Florenzano, R., Serrano, T., Roizblatt, A., Rodríguez, J. Familia, Factores Protectores, Resiliencia, Conductas de Riesgo Adolescente: Relación con el Desarrollo Yoico. Boletín de Investigaciones en Educación, Facultad de Educación, Pontificia Universidad Católica de Chile, pgs. 131-150, 1999

24 Blum R y Cols. Minnesota Adolescent Health Survey. Departamento de Pediatría, Universidad de Minnesota, 1992

Valdés, M., Serrano., T., Rodríguez, J., Roizblatt, A., Florenzano, R., Labra, J.F. Características del Funcionamiento familiar que predicen conductas de riesgo en adolescentes y sus familias. Cuadernos Médico-Sociales, 38: 14-21, 1997.

26 Perry JP, Herman JL y Van der Kolk, BA. The Traumatic Antecedents Inverview, Universidad de Harvard, Cambridge, 1992

27 Fonagy, P. y Target, M. Psychoanalytic Theories: Perspectives from Developmental Psychopathology. Brunner-Routledge, Nueva York, 2003. 
